administration will restore the olfactory sensation of patients with narcolepsy. The authors comment that orexin A intranasally is, theoretically, a promising treatment for narcolepsy and may be considered for future trial.

\title{
DIAGNOSIS AND MANAGEMENT OF NARCOLEPSY REVIEWED
}

Researchers at Duke University Medical Center, and Veterans Affairs Medical Center, Durham, NC, review the epidemiology, pathophysiology, diagnosis, and treatment of pediatric narcolepsy. Narcolepsy is a disorder of rapid eye movement (REM) sleep characterized by excessive daytime somnolence, associated with sleep paralysis, hypnagogic (when falling asleep) and hypnopompic (when awakening) hallucinations, and cataplexy. Prevalence is $0.05 \%$ in the US and Europe; $0.18 \%$ in Japan; and $0.002 \%$ in Israel; greater in males than females. Onset highest in second decade, with peaks at 14 yrs and 35 yrs. Etiology is unknown, possible neurodegenerative with autoimmune component. Patients with narcolepsy and cataplexy share the same HLA genotype. Predominantly sporadic, sometimes familial, only $25-31 \%$ concordance in twin studies. CSF levels of hypocretins less than 110 $\mathrm{pg} / \mathrm{ml}$ are diagnostic of narcolepsy with cataplexy. Obesity with narcolepsy is associated with low $24 \mathrm{hr}$ leptin levels, a hormone secreted by adipose tissue. Narcolepsy is idiopathic or secondary (symptomatic) and caused by hypothalamic tumors, head trauma, multiple sclerosis, vascular, and encephalitic disorders. In addition to the classic tetrad of narcolepsy symptoms, semi-purposeful, automatic behavior is common during the day, sometimes misdiagnosed as epilepsy, and frequent noctural awakenings. Obesity and obstructive sleep apnea frequently coexist. The interval between symptom-onset to diagnosis is about 10 years. Misdiagnosis is common in children with narcolepsy, leading to delay in treatment. The history combined with polysomnography and mean sleep latency (MSLT) $<8$ minutes are used in diagnosis in adults and in children older than 8 years. Two or more sleep onset REM sleep periods (SOREMP) in a MSLT within $15 \mathrm{~min}$ of sleep onset are consistent with narcolepsy. MRI is normal in idiopathic narcolepsy. Treatment includes lifestyle changes, brief naps, caffeine, reduced carbohydrates or Atkins diet, and pharmacotherapy (methylphenidate, amphetamines, and non-approved pediatric use of modafinil (Provigil), and sodium oxybate (Xyrem). Sodium oxybate is particularly effective in cataplexy in adults. Antidepressants have also been used to treat cataplexy and hypnogogic hallucinations in adults. (Peterson PC, Husain AM. Pediatric narcolepsy. Brain Dev Nov 2008;30:609-623). (Respond: A.M. Husain, E-mail: Aatif.husain@duke.edu )

COMMENT. A high index of suspicion is required in the diagnosis of narcolepsy. This review provides an excellent account of the diagnosis and treatment of narcolepsy. A sleep specialist reports the highest success rate for correct diagnosis among neurologists $(55 \%)$, psychiatrists $(11 \%)$, and pediatricians (0\%). (Kryger MH et al. Sleep 2002;25:36-41).

From the Archives: Idiopathic narcolepsy: a disease sui generis. By Adie WI. Brain 1926;49:257-306 and The narcolepsies. By Kinnear Wilson SA. Brain 1928;51:63-109. Adie describes cases seen at Queen Square and misdiagnosed as epilepsy, and summarizes earlier reports by Gelineau (1880). Examples of patients are two soldiers courts-martialed for falling asleep on listening-post duty. Adie coins the term cataplexy associated with narcolepsy. He considers the etiology an endocrine-nervous disorder, a disease sui generis, a proposal 
dismissed by his senior colleague, Kinnear Wilson, who regards narcolepsy as a syndrome with several different causes, traumatic, endocrine, epileptic, toxi-infective, circulatory, tumor, and idiopathic. Neither Adie nor Kinnear Wilson refers to hyposmia in their account of narcolepsy-cataplexy disorder, but they did locate the pathology in the floor of the third ventricle in symptomatic cases. (Compston A. Brain Oct 2008;131:2532-2535).

\section{ELECTROENCEPHALOGRAPHY}

\section{PROGNOSTIC VALUE OF EEG IN ASPHYXIATED NEWBORNS TREATED WITH HYPOTHERMIA}

Researchers at Children's Hospitals in Milan, Italy, determined the prognostic value of electroencephalographic patterns in 23 newborns with severe perinatal hypoxic-ischemic encephalopathy, treated with hypothermia. EEG monitoring was obtained within 48 hours after birth, and at follow-up at ages 1 week, 1 month, 3-6 months, and 1 year. EEG background activity was classified as follows: 1) inactive pattern; 2) severe low-voltage continuous pattern; 3) trace alternant-like, discontinuous pattern; and 4) monomorphic middle-voltage, continuous $30-100 \mathrm{mcV}$ activity, with poor spatial and sleep-state organization. Pattern 1 (inactive) in the first 48 hrs was associated with death or severe neurologic sequelae. Pattern 2 (low-voltage continuous) at age 1 week indicated a poor prognosis, and the persistence of EEG abnormalities in $67 \%$ patients at age 1 month was associated with a higher risk of neurologic sequelae. A normal EEG at age 1 month was associated with a favorable outcome at age 1 year. After 1 month of age, the EEG is less sensitive but more specific in prediction of outcome, due to the natural trend toward normalization with age. At age 1 year, $52 \%$ infants had normal neurologic examinations, $13 \%$ had minor sequelae, and $17 \%$ major sequelae; $17 \%$ had died within 1 month of age. (Mariani E, Scelsa B, Pogliani L, Introvini P, Lista G. Prognostic value of electroencephalograms in asphyxiated newborns treated with hypothermia. Pediatr Neurol Nov 2008;39:317-324). (Respond: Dr Scelsa, Department of Child Neurology, Vittori Buzzi Children's Hospital, Via Castelvetro 32, 20154 Milan, Italy. E-mail: b.scelsa@icp.mi.it).

COMMENT. These results confirm previous findings that background EEG abnormalities detected in newborns soon after hypoxic-ischemic encephalopathy are predictive of outcome, even in patients treated with hypothermia.

\section{AMPLITUDE-INTEGRATED EEG IN THE NEWBORN}

Th value of amplitude-integrated electroencephalography (aEEG) in the newborn is explored by researchers at Washington University, St Louis; Wilhelmina Children's Hospital, Utrecht, Netherlands; and Uppsala University Hospital, Sweden. The system was originally designed to monitor lower amplitude signals of 1 to $10 \mathrm{mcV}$ and depressed cerebral activity in adults undergoing bypass surgery, as well as seizure activity. Meta-analysis has confirmed that the aEEG pattern in the first 6 hours of life of term newborns with hypoxic-ischemic encephalopathy is strongly predictive of outcome. Pattern-recognition may be more reliable than amplitude in the evaluation of aEEG. The electrode placement over parietal areas, 